\title{
An Algorithm for GPS Anti-Jamming Based on Improved FastICA
}

\author{
Xiao-Bo Liao* \\ University of Electronic Science and Technology of China, Research Institute of Electronic Science and Technology, \\ Sichuan 611731, China
}

\begin{abstract}
In the practical environment, it is difficult for the user receiver to get DOA information of GPS signals in advance. In this paper, on the basis of blind source separation (BSS) theory, a signal-separation-based GPS antiinterference algorithm has been proposed. In accordance with BSS process modes, wavelet denoising algorithm is first adopted to restrain the influence of noise on separation algorithm. Then, improved FastICA separation algorithm is adopted to separate the mixed signals. Finally, secondary wavelet denoising algorithm is used to restrain the amplified noise and GPS signal anti-interference is realized. The test shows that: the anti-interference algorithm proposed in this paper is effective. Under the same SNR, the algorithm has better anti-interference effect than traditional separation.
\end{abstract}

Keywords: Anti-jamming, BSS, DOA, FastICA, Wavelet de-noising.

\section{INTRODUCTION}

Global Positioning System (GPS) is a system which works out the relative distance between the carrier and satellite through radio signals transmitted by the navigational satellite. Then based on the satellite's known position relative to the ground, it calculates and confirms the carrier's position on the earth. With the fast development of science and technology, GPS has been more and more extensively applied to military and civil fields. However, GPS receiver is vulnerable to various interferences, which reduces the positioning and navigation performances. In worse cases, the receiver may not function properly. So, it is urgent to improve the anti-interference capability of GPS receiver. With the increasing complexity of interference signal modes, many anti-interference processing methods have been designed [1], such as linear constrained minimum variance (LCMV) of airspace anti-interference technology, minimum variance distortionless response (MVDR), etc. These technologies can play certain anti-interference function to some extent. However, they all require the recipients to know beforehand the direction of arrival (DOA) of each signal $[2,3]$. In the actual working environment of the receiver, the recipient can not get the prior information of DOA in advance. This is an obvious defect of traditional anti-interference technologies. Blind source separation (BSS) technology can solve the problem very well and provide new idea and method for GPS anti-interference.

In recent years, BSS technology has become a hot topic of signal processing research. In particular, since Herault and Jutten put forward neural-like BSS technology in 1991, many BSS algorithms have been proposed.

In 1994, P. Comon expanded principal component analysis and expounded the concept of independent component

\footnotetext{
*Address correspondence to this author at the University of Electronic Science and Technology of China, Research Institute of Electronic Science and Technology, Sichuan 611731, China; Tel: 18986139113 ; E-mail: hunter2011@foxmail.com
}

analysis (ICA). In 1995, Bell and Sejnowski classified BSS into the framework of information theory, and combined information theory and ICA into a unified framework. In 1997, Havarinen and Oja studied batch algorithm and put forward fixed-point FastICA algorithm based on Newton's iteration method. This laid solid foundation for the practical application of BSS [3]. In 1996, Zhang Xianda introduced the theoretical basis of BSS and set forth relevant algorithms. His research was a prelude for the domestic study of BSS. Of the existing ICA algorithms [4-6], most algorithms are based on the assumption of noise free condition. But in the actual environment, observed signals are often mixed with various noises. If the noise is not negligible, these algorithms cannot obtain ideal separation signals. In this paper, we analyze the influence of noise on separated signals, introduce secondary denoising processing [7], and restrain the influence of noise on separation algorithm $[7,8]$. By introducing descending factor, we reduce the dependency of separation matrix on the initial value, effectively separate GPS signals from interference signals, and improve the anti-interference capability of the algorithm. In accordance with the basic theory and application of BSS, we present the BSS-based GPS antiinterference algorithm [9].

The structure of the paper is like this: part 2 introduces signal models and observed signal models; part 3 introduces the anti-interference algorithm and its performance evaluation in detail; part 4 is test result and analysis; and the last part is conclusion.

\section{SIGNAL MODELS}

The GPS signals transmitted by a satellite include 3 components: carrier wave, spreading code and data code. GPS is a CDMA-based spread-spectrum communication (SSC) system. The spreading code in this paper is $\mathrm{C} / \mathrm{A}$ code, and the period is $1 \mathrm{~ms}$. As a data code consists of $20 \mathrm{C} / \mathrm{A}$ code periods, so in the record of $1 \mathrm{~ms}$ data, the occurrence of $\mathrm{C} / \mathrm{A}$ code phase jump caused by data code is low. In this paper, data code is not modulated to $\mathrm{C} / \mathrm{A}$ code. Instead, C/A 
code is directly modulated to carrier wave. The expression of the GPS signal model in this paper is:

$$
s_{1}(t)=A x(t-\tau) \sin \left(2 \pi\left(f_{0}-f_{d}\right)(t-\tau)+\theta\right)
$$

In which, $\mathrm{A}$ is the average amplitude of satellite signals received by the antenna, $x(t)$ is the $\mathrm{C} / \mathrm{A}$ code broadcast by the satellite, $\theta$ is the initial phase of carrier wave, $\tau$ is signal propagation delay, $f_{d}$ is the Doppler shift of signals and $f_{0}$ is the IF carrier frequency.

At present, there are mainly two kinds of interference to GPS signals: blanket interference and deception interference. Only with the coordination of blanket interference can deception interference avoid being recognized by the opponent. So, US army believes the major threat for the military GPS receiver is blanket interference. In this paper, we mainly discuss blanket interference. The common interference signals are:

Single-tone continuous wave interference:

$s_{2}(t)=A \cos \left(w_{\Delta} t T_{c}+\theta\right)$

In which, $A$ is the amplitude of the interference signals, $w_{\Delta}$ is the excursion of spread-spectrum signal from the center frequency, $T_{c}$ is chip width, and $\theta$ is within the random phase of $[0,2 \pi)$.

Sweeping frequency interference:

$s_{3}(t)=A \cos \left(2 \pi f_{0} t+\pi \mu_{0} t^{2}+\theta\right)$

In which, $\mathrm{A}$ is the amplitude of the interference signals, $\theta$ is the initial phase of the interference signals, $f_{0}$ is IF carrier frequency and $\mu_{0}$ is sweeping frequency speed.

Sawtooth wave FM interference:

$s_{4}(t)=A \cos \left(2 \pi f_{0} t+\int_{0}^{t} K v(t) d t+\theta\right)$

In which, $\mathrm{A}$ is the amplitude of the interference signals, $f_{0}$ is IF carrier frequency, $\theta$ is the initial phase of the interference signals, and $\mathrm{K}$ is FM transconductance.

Sawtooth wave signals:

$v(t)=A\left[t u(t)-\sum_{n=1}^{\infty} u(t-n T) \cdot T\right]$

In accordance the present BSS theories [10], we assume there are 4 source signals (including GPS signal and interference signal), and there are 4 reception channels in the user's receiver. In the course of transmission, the signals mix instantaneously and the receiver system is linear time invariant. Then, the observed signals in the receiver system at $t$ moment can be expressed as:

$$
X(t)=A \bullet S(t)+n(t)
$$

In which, $X(t)$ is the vector of observed signals, $\mathrm{A}$ is the mixture matrix in the transmission, $S(t)$ is the vector of the source signals and $\mathrm{n}(t)$ is additive noise signals. For the antiinterference algorithm put forward in the paper, we also assume that various signals are independent of each other and independent of noise.

At present, wavelet threshold denoising is fast and effective in suppressing noise [10]. In this paper, we adopt wavelet threshold denoising method to conduct denoising processing to GPS signal. After denoising processing, the SNR of received mixed-signals can be enhanced. Meanwhile, some useful detail signals might get lost. So, in the early denoising processing, the source signals should be retained to the greatest extent. As the early denoising is incomplete, the obtained signals are not pure source signals. After separation algorithm, the additive noise can be amplified. Suppose the separated signal is $Y^{\prime}$, and the separation matrix derived from separation algorithm $W$, then:

$$
Y^{\prime}=W \cdot X^{\prime \prime}=W \cdot\left[A S(t)+n^{\prime}(t)\right]=W \cdot A S(t)+W \cdot n^{\prime}(t)
$$

$n^{\prime}(t)$ is the few residual noise after early denoising. After separation matrix $W$, the noise variance can be amplified. So it is necessary to conduct secondary denoising to the separated noise so as to improve the SNR of processed GPS signals.

In the course of BSS, we often need to make assumptions for the mixed signals, e.g. zero mean value of observed signals, full rank of the mixture matrix, etc. But in most cases of the actual application, neither observed signals nor source signals can meet all the assumptions. Meanwhile, to reduce the complexity of BSS calculation, we need to conduct pretreatment to observed mixed signals: centralization and whitening. The pretreatment can improve the subsequent convergence rate and stability of separation algorithm.

Central-limit theorem (a kind of statistical knowledge) shows that: the non-gaussianity of source signals is higher than that of observed signals. So we can regard nongaussianity as a criterion of signal separation [8].

Negentropy can quantitatively measure the nongaussianity of random signals. In this paper, we create cost function through non-gaussianity. Among probability density functions with the same covariance matrix, Gaussian distribution has the highest entropy, so the KL divergence between arbitrary probability density function $p(x)$ and Gaussian distribution $p_{G}(x)$ with the same covariance matrix is often used to measure the non-gaussianity of the probability density function $p(x)$, and it is called negentropy. It is expressed as follows:

$J[x]=J[p(x)]=K L\left[p(x), p_{G}(x)\right]$ 
$=\int p(x) \log \left[\frac{p(x)}{p_{G}(x)}\right] d x=H_{G}(x)-H(x)$

The probability theory proves that negentropy is a reasonable measure of non-gaussianity. If non-gaussianity is estimated in strict accordance with the definition, then it needs the estimation form of probability density function of the source signals. But in real work, this is very hard. So the approximate expression of negentropy is very important. The negentropy approximate expression based on maximum entropy principle is:

$J(Y) \approx C \cdot[E\{G(Y)\}-E\{G(V)\}]^{2}$

In which, $Y=W \cdot X, Y=\left(y_{1}, y_{2}, y_{3}, y_{4}\right)^{T}$ is the estimation of the source signals, $W=\left(w_{1}, w_{2}, w_{3}, w_{4}\right)$ is separation matrix, $\mathrm{C}$ is a positive constant, $\mathrm{V}$ is the Gaussian variable of zero mean value and unit variance. $G(x)=\frac{1}{a} \log \cosh (a x)$ is non-quadratic function, and $a \in[1,2]$ 。

The negentropy expression of non-gaussianity is created above. To find out the projection direction of $y=W^{T} x$, we need to get maximum value through the formula below:

$J_{G}(y)=c[E\{G(y)\}-E\{G(v)\}]^{2}$

In which, $v$ is the standard Gaussian distribution variable of $y=W^{T} x$ with the same mean value and covariance matrix. The purpose of signal separation is to find a proper $\mathrm{W}$ and get a maximum value of $J_{G}(y)$. In this way, the maximization above can be translated into optimization of $E\left\{G W^{T} x\right\}$.

In accordance with Kuhn-Tucker condition and subject to the constraint condition of $E\left\{\left(W^{T} x\right)^{2}\right\}=\|W\|^{2}=1$, the optimal solution to negentropy-based cost function can satisfy the following Lagrange formula:

$E\left[x g\left(W^{T} x\right)\right]-\beta W=0$

In which, $\beta$ is a constant, $g(\bullet)$ is the derivative of $G(\bullet)$. When $W_{0}$ is the optimal value of $W$, we can get:

$\beta=E\left[W_{0}^{T} x g\left(W_{0}^{T} x\right)\right]$

In this way, the approximate value of iteration can be obtained.

The above equation can be solved with newton method. Suppose the left function of the equation is F, then its Jacobi matrix is:

$$
J[F(W)]=E\left[x x^{T} g^{\prime}\left(W^{T} x\right)\right]-\beta I
$$

As the observation data $\mathrm{x}$ has been whitened, then the first item of Jacobi can be simplified as:

$$
E\left[x x^{T} g^{\prime}\left(W^{T x}\right)\right] \approx E\left[x x^{T}\right] E\left[g^{\prime}\left(W^{T} x\right)\right]=E\left[g^{\prime}\left(W^{T} x\right)\right] I
$$

So, the Jacobi matrix becomes diagonal matrix, and its inversion operation is simplified. $\beta$ can be approximated as $\beta=E\left[W^{T} x g\left(W^{T} x\right)\right]$, then we can get an approximate Newton's iteration method:

$$
W=W-\left\{E\left[x g\left(W^{T} x\right)-\beta W\right]\right\} /\left\{E\left[x g\left(W^{T} x\right)-\beta\right]\right\}
$$

After simplification, we get:

$$
\left\{\begin{array}{c}
W^{+}=E\left\{x g\left(W^{T} x\right)\right\}-E\left\{g^{\prime}\left(W^{T} x\right)\right\} W \\
W^{*}=W^{+} /\left\|W^{+}\right\|
\end{array}\right.
$$

In this paper, descending factor is introduced through Newton's iteration method. It reduces the algorithm's early dependence on separation matrix value and enhances the algorithm's separation effect. From the algorithm above we know that, iteration point can be expressed as: $W_{k+1}=W_{k}+\Delta W$. If we choose variable-step descending factor $b(n)$, and make:

$W_{k+1}=W_{k}+b(n) \Delta W$

A new iterative formula can be obtained:

$W_{k+1}=W_{k}-b(n) E\left\{x g\left(W_{k}^{T} x\right)\right\} / E\left\{g^{\prime}\left(W_{k}^{T} x\right)\right\}$

In which, $b(n)$ is descending coefficient or forgetting factor. When $b(n)=1$, the algorithm is identical with original FastICA algorithm, $b(n) \in(0,1)$,

From the above, the iterative formula of the improved algorithm is:

$$
\left\{\begin{array}{c}
W_{k}^{0}=W_{k} \\
W_{k}^{i}=E\left(x g\left(W_{k}^{i-1} x\right)\right)-b(i) E\left(g^{\prime}\left(W_{k}^{0} x\right)\right) W \quad, \quad i=1,2, \cdots, m \\
W_{k+1}=W_{k}^{m}
\end{array}\right.
$$

The anti-interference algorithm presented in this paper is based on BSS algorithm, so the anti-interference performance is evaluated through separation effect. To evaluate the separation effect of blind source signal separation algorithm, the similarity coefficient of GPS signal estimation and GPS source signal is set as the performance index. The similarity coefficient is expressed as:

$$
\rho=\frac{\operatorname{cov}(s(t), y(t))}{\sqrt{\operatorname{cov}(s(t), s(t)) \operatorname{cov}(y(t), y(t))}}
$$

In which, $s(t)$ is the source signal, $y(t)$ is the estimation of the source signal. When $s(t)$ and $y(t)$ are 
independent of each other, $|\rho| \rightarrow 0$; when $s(t)$ is a better estimation of $y(t),|\rho| \rightarrow 1$ 。

Crosstalk error is the deviation between composite matrix $C=W H$ and the general switch matrix (each line and row only consist of element 1). Crosstalk error is used to measure the separation performance of the algorithm. Its definition formula is:

$E=\sum_{i=1}^{N}\left(\sum_{j=1}^{N} \frac{c_{i j}}{\max \left|c_{i j}\right|}-1\right)+\sum_{j=1}^{N}\left(\sum_{i=1}^{N} \frac{c_{i j}}{\max \left|c_{i j}\right|}-1\right)$

When the composite matrix $C$ is approximate to switch matrix, the value of the index will be smaller, and the crosstalk Error will be smaller too. It indicates that the algorithm achieves good separation effect. On the contrary, when the composite matrix $C$ deviates from the switch matrix, the value of the index will be bigger, meaning the algorithm achieves poor separation effect.

\section{SIMULATION}

We conduct noise-free separation test and noise separation test on four-channel signals (signal model as described in part 2). Suppose the receiver is a 4-channel linear antenna array, the distance of adjacent antennas is half-wavelength and the duration of received data sample is $1 \mathrm{~ms}$. The central carrier frequency of the GPS is $f_{0}=3.069 \mathrm{MHz}$, Doppler shift $f_{d}=-3 \mathrm{kHz}$, code phase $\tau=600$, and the frequency of interference signals is identical with the central carrier frequency of the GPS. In the LCMV algorithm test (contrast test), we suppose the DOA of GPS and interference signals are respectively: $-20^{\circ}, 0^{\circ}\left(10^{\circ}\right) 40^{\circ}$. Below is the test simulation result.

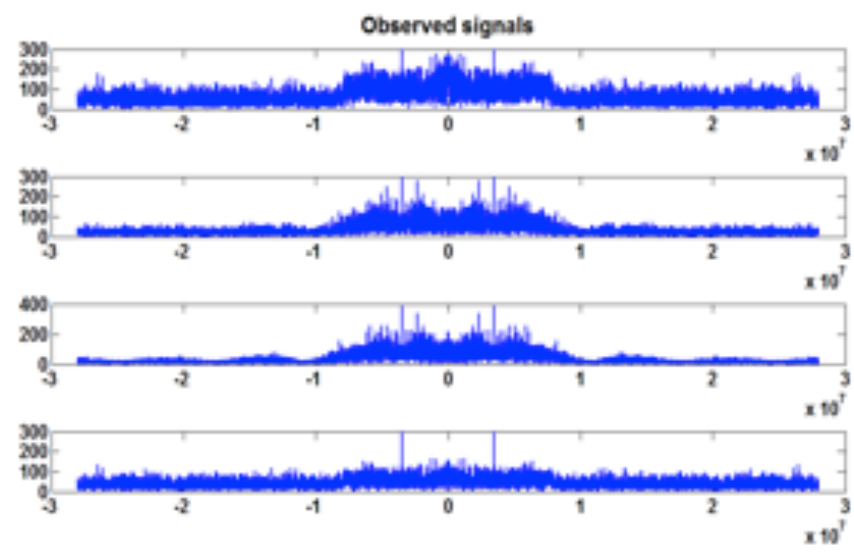

Fig. (1). Observed signals.

We conduct two contrast tests to verify the feasibility of the anti-interference methods presented in the paper. Test A adopts the method in this paper to implement GPS antiinterference. Test B adopts traditional LCMV method to implement GPS anti-interference. The simulation condition is described as above, and the simulation result is presented in the figure.
Fig. (1) shows the frequency spectrum of the observed signals. It indicates that the frequency spectrum of source signals seriously overlaps. Traditional filter anti-interference can hardly separate the GPS signals.

Figs. $(2,3)$ respectively show the frequency spectrum of the source signals and separated signals. Fig. (3) indicates that the signals totally overlap in the course of transmission. Fig. (4) indicates that the frequency spectrograms of corresponding separated signals and source signals are consistent. It means accurate GPS can be obtained with the method in this paper, and the signal-separation-based GPS anti-interference is feasible.

Table 1. Similarity coefficients.

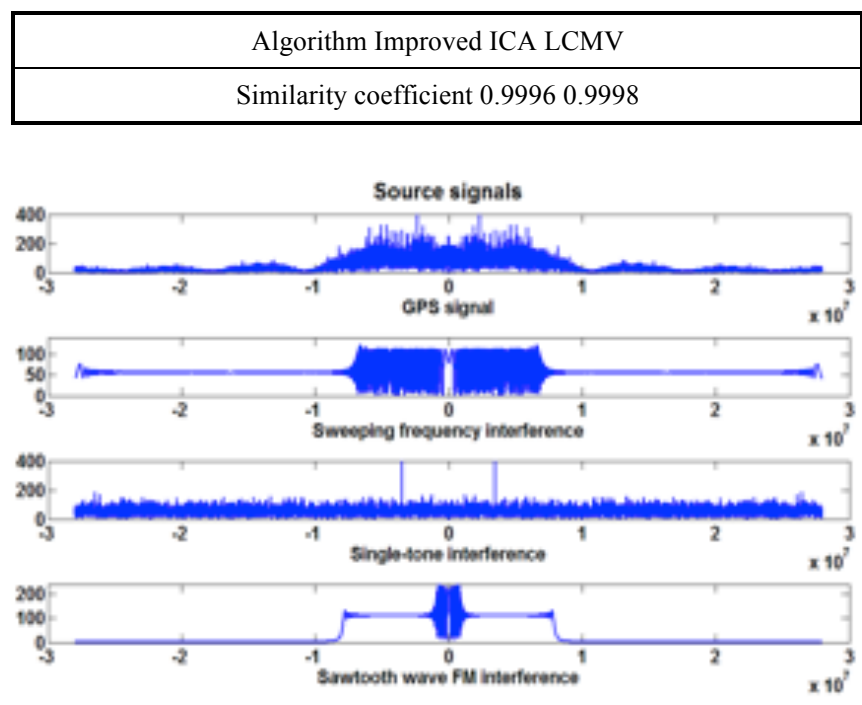

Fig. (2). Source signals.

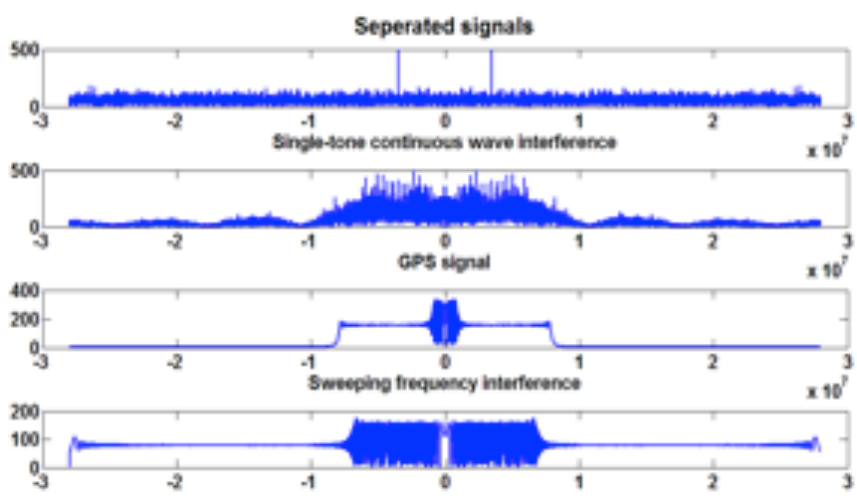

Fig. (3). Separated signals.

The similarity coefficients of GPSs obtained with the method in this paper and the LCMV method are set forth in Table 1. $\rho_{\text {ICA }}$ and $\rho_{\text {LCMV }}$ are close to 1 . The contrast of GPS signal spectrum estimation and GPS source signal spectrum is shown in Fig. (4). The GPS signal spectrum estimation and GPS source signal spectrum obtained with the two methods are identical. The comparison between time-domain similarity coefficients and frequency spectrograms suggest that, under the condition of unknown DOA of signals, with the method presented in this paper, GPS signal estimation 

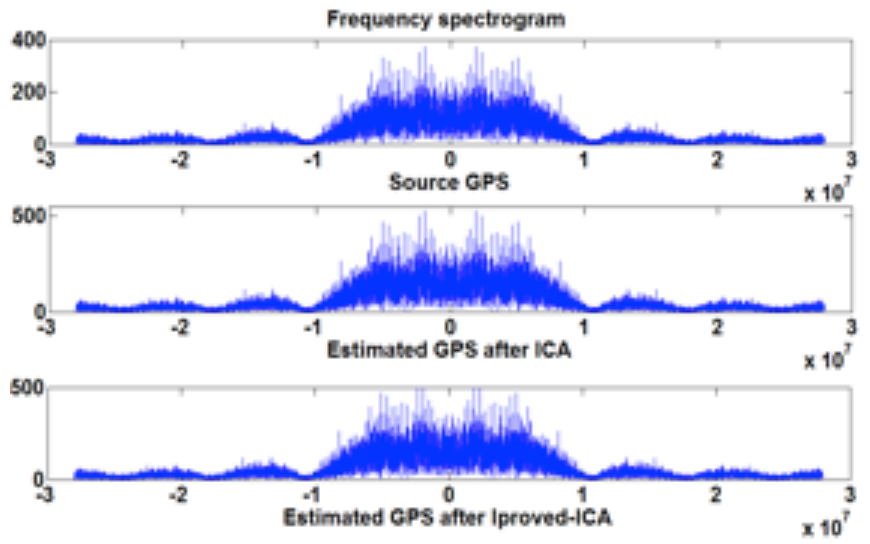

Fig. (4). Frequency spectrogram.

can be obtained through separation and the separation effect is better than that of LCMV algorithm. It shows the separation algorithm presented in this paper is more practical for GPS anti-interference. It reduces the requirement of user's receiver for the DOA of GPS signals and interference signals.

The contrast tests also evaluate the influence of additive noise and the anti-noise capacity of the algorithm. Test A adopts traditional anti-noise algorithm and test $\mathrm{B}$ adopts the anti-noise algorithm of this paper. The simulation result is shown in the figure below.

Table 2. Similarity coefficients with different SNR.

\begin{tabular}{|c|c|c|c|c|c|}
\hline SNR & 30dB & 20dB & $\mathbf{1 0 d B}$ & $\mathbf{5 d B}$ & $\mathbf{0 d B}$ \\
\hline \hline$\rho_{\text {Impoved-ICA }}$ & 0.9961 & 0.9829 & 0.8218 & 0.7764 & 0.7374 \\
\hline$\rho_{\text {ICA }}$ & 0.9923 & 0.9617 & 0.7589 & 0.6957 & 0.5932 \\
\hline
\end{tabular}

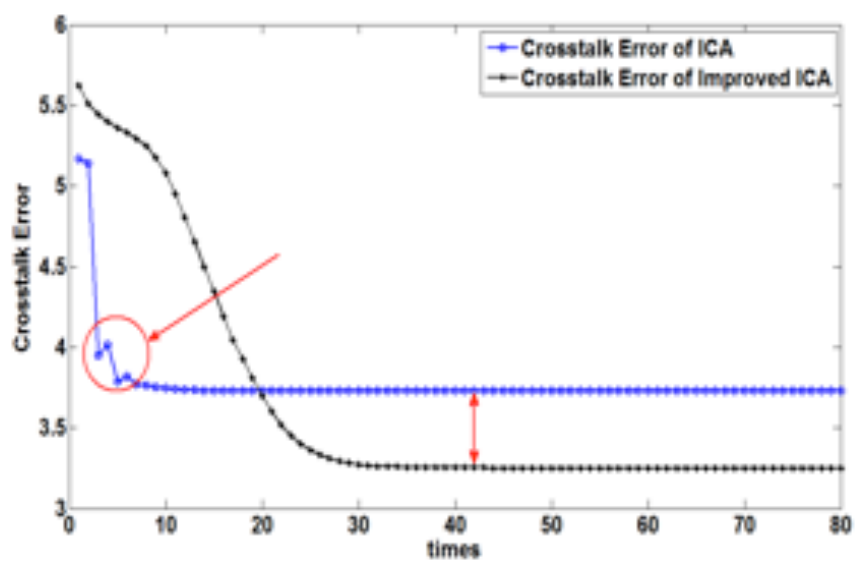

Fig. (5). The correlation curves of crosstalk errors.

The similarity coefficients of the two tests under different SNR are summarized in Table 2. With high SNR, the similarity coefficients of the two algorithms are both close to 1. With the decrease of SNR, the similarity coefficient of the algorithm described in this paper is more close to 1 , while the similarity coefficient of traditional algorithm declines sharply. It means the algorithm described in this paper has a stronger anti-noise capacity. When $\mathrm{SNR}=20 \mathrm{~dB}$, the correlation curves of crosstalk errors derived with the two algorithms is shown in Fig. (5). The correlation curve of crosstalk error derived with the algorithm presented in this paper is smoother than that derived with traditional algorithm. This indicates that the algorithm presented in this paper can process calmly in the iteration. The red mark in Fig. (5) shows instability occurs in the iteration of traditional separation algorithm, and the separation effect is influenced. Under stable iteration, the crosstalk error derived with the algorithm presented in this paper is much smaller than that derived with traditional algorithm, meaning the separated signals with the algorithm presented in this paper is a better estimation of the source signals. Fig. (6) shows that the PI curves of both algorithms decline with the rise of SNR. The separation effect of the algorithm proposed in this paper is much better than that of traditional algorithm. With the increase of SNR, both PIs approach to 0. With SNR=20dB, the PI of the algorithm proposed in this paper is quite small, and achieves good separation effect. The comparison of curves in Fig. (6) shows that, with equal SNR, the algorithm proposed in this paper has better separation effect and stronger anti-interference capability. This means under noise environment, the algorithm proposed in this paper has better anti-interference effect.

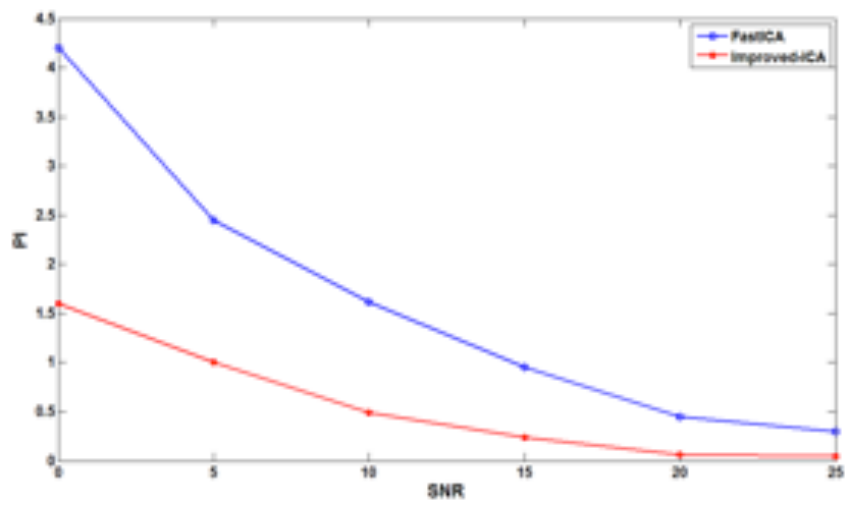

Fig. (6). The correlation curves of crosstalk errors.

The test simulation shows, under both noise-free and noise environment, the algorithm proposed in this paper can separate GPS signals from interference signals, obtain good GPS signal estimation, and realize GPS anti-interference. Noise imposes an unnegligible influence on the separation effect. To improve the anti-noise capability of signal, separation-based anti-interference algorithm is a field worth researching.

\section{CONCLUSION}

GPS is the most widely used positioning system in today's world. Its biggest weakness is the vulnerability to interference, so the study of GPS anti-interference technology is of great significance. The algorithm presented in this paper can separate GPS signals from interference signals, obtain good GPS signal estimation, and realize GPS anti-interference. Noise imposes an unnegligible influence on the separation effect. The optimized algorithm in this paper enhances the anti-noise capability of the separation 
method. It is significant to study the anti-noise capability of the separation method and it can further promote the application of BSS algorithm to GPS anti-interference.

\section{CONFLICT OF INTEREST}

The author confirms that this article content has no conflict of interest.

\section{ACKNOWLEDGEMENTS}

This work is supported by the Key Project of Guangxi Social Sciences, China (No. gxsk201424), the Education Science fund of the Education Department of Guangxi, China (No. 2014JGA268), and Guangxi Office for Education Sciences Planning, China (No. 2013C108).

\section{REFERENCES}

[1] H. Hu and N. Wei, "A study of GPS jamming and anti-jamming," In: $2^{\text {nd }}$ International Conference on Power Electronics and Intelligent Transportation System (PEITS), 2009, pp. 388-391.

[2] S.H.I. Wensen, Z.H.U. Hai, and C.A.I. Peng, "Research on DOA estimation of GPS based on the C/A code cross-correlation characteristic of the signal", Journal of Projectiles, Rockets, Missiles and Guidance, vol. 33, no. 6, pp.189-192, 2013.
[3] U. Ahmed, M.T. Islam, and M. Ismail, "Estimating DoA from radio frequency RSSI measurements using multi-element femtocell configuration," Sensors Journal, IEEE, vol. 15, pp. 2087-2092, 2015.

[4] Y.-b. Quan, J.-f. Wang, Y. Yue, L. Li, and J. Yan, "Present situation and development trend of blind source separation," Communications Technology, vol. 4, p. 5, 2011.

[5] V. Zarzoso and P. Comon, "Robust independent component analysis by iterative maximization of the kurtosis contrast with algebraic optimal step size", In: IEEE Transactions on Neural Networks, vol. 21, pp. 248-261, 2010.

[6] C. W. Hesse and C.J. James, "The FastICA algorithm with spatial constraints," Signal Processing Letters, IEEE, vol. 12, pp. 792-795, 2005.

[7] A. Paraschiv-Ionescu, C. Jutten, K. Aminian, B. Najafi, and P. Robert, "Wavelet de-noising for highly noisy source separation," In: IEEE International Symposium on Circuits and Systems, 2002, pp. I-201.

[8] N. El-Sheimy, S. Nassar, and A. Noureldin, "Wavelet de-noising for IMU alignment," Aerospace and Electronic Systems Magazine, IEEE, vol. 19, pp. 32-39, 2004.

[9] X. Kun-lai, L. Zheng, and J. Wen-li, "Broadband blind source separation algorithm based on array model," Systems Engineering and Electronics, vol. 4, p. 002, 2014.

[10] X.-x. Chen, Y.-j. Wang, and L. Liu, "Deep study on wavelet threshold method for image noise removing," Laser \& Infrared, vol. 42, pp. 105-110, 2012.

(C) Xiao-Bo Liao; Licensee Bentham Open.

This is an open access article licensed under the terms of the (https://creativecommons.org/licenses/by/4.0/legalcode), which permits unrestricted, non-commercial use, distribution and reproduction in any medium, provided the work is properly cited. 